ARTIGOS

Submetido 14.07.2020. Aprovado 10.05.2021

Avaliado pelo sistema double blind review. Editora Científica: Andrea Lago da Silva

Versão original | DOI: http://dx.doi.org/10.1590/So034-759020220204

\title{
DANO AMBIENTAL: QUANDO A RAIVA PODE LEVAR À DESCONTINUIDADE DO FORNECEDOR
}

\author{
Environmental damage: When anger can lead to supplier discontinuity \\ Daño ambiental: Cuando el enojo puede conducir a la discontinuidad del proveedor
}

Josefer de Lima Souza ${ }^{1}$ | joseferlima@hotmail.com | ORCID: 0000-0002-2071-2393

Vilmar Antonio Gonçalves Tondolo² | vtondolo@gmail.com | ORCID: 0000-0002-3116-2585

Rosana da Rosa Portella Tondolo² ${ }^{2}$ rosanatondolo@gmail.com | ORCID: 0000-0002-2410-8038

Guillherme Lerch Lunardi' ${ }^{1}$ | gllunardi@furg.br | ORCID: 0000-0003-3250-2796

Flávio Régio Brambilla3 | flaviobrambilla@terra.com.br | ORCID: 0000-0002-9398-7240

${ }^{1}$ Universidade Federal do Rio Grande, Programa de Pós-Graduação em Administração, Rio Grande, Rio Grande do Sul, Brasil

${ }^{2}$ Universidade Federal de Pelotas, Centro de Ciências Socio-Organizacionais, Pelotas, Rio Grande do Sul, Brasil

3Universidade de Santa Cruz do Sul, Programa de Pós-Graduação em Administração, Santa Cruz do Sul, Rio Grande do Sul, Brasil

\section{RESUMO}

O estudo analisou os efeitos diretos e indiretos da controlabilidade e da responsabilidade na descontinuidade do fornecedor após dano ambiental. Os dados foram coletados junto a 267 indivíduos com experiência em gestão, por meio de um experimento baseado em cenários. Os resultados indicaram que a controlabilidade influencia a descontinuidade do fornecedor, bem como anger do comprador, quando o fornecedor, e não a natureza, possui controle sobre o dano ambiental ocorrido. 0 efeito indireto da controlabilidade foi parcialmente explicado pela anger sentida. Já os efeitos direto e indireto da responsabilidade não mostrou significância, sendo parcialmente explicado de maneira moderadora na relação entre a controlabilidade e a descontinuidade do fornecedor após o dano ambiental. O estudo identifica o papel comportamental de uma emoção negativa experimentada durante crises gerenciais, influenciando as tomadas de decisões de indivíduos quanto à descontinuidade do fornecedor após a ocorrência de um dano ambiental.

PALAVRAS-CHAVE | Compras sustentáveis, descontinuidade do fornecedor, controlabilidade, responsabilidade do fornecedor, experimento.

\section{ABSTRACT}

This study analyzed the direct and indirect effects of controllability and responsibility on supplier discontinuity following environmental damage. Data were collected through a scenario-based experiment from 267 individuals with management experience. The results indicated that controllability has an influence on supplier discontinuity, as does anger (a negative emotion), when the supplier, rather than nature, has control over the environmental damage caused. The indirect effect of controllability was partially explained by anger. The direct and indirect effects of responsibility, on the other hand, were not significant, and were partially explained in a moderating role in the relationship between controllability and the non-retention of suppliers following environmental damage. The study contributes by identifying the behavioral role of the negative emotion that is experienced during management crises, thus having an influence on the decision making of individuals that is related to the discontinuity of suppliers following environmental damage.

KEYWORDS I Green purchasing, supplier discontinuity, controllability, supplier responsibility, experiment.

\section{RESUMEN}

El estudio analizó los efectos directos e indirectos de la controlabilidad y la responsabilidad sobre la discontinuidad del proveedor después del daño ambiental. Los datos fueron recolectados de 267 personas con experiencia en gestión, a través de un experimento basado en escenarios. Los resultados indicaron que la controlabilidad influye en la discontinuidad del proveedor así como la ira del comprador cuando el proveedor, y no la naturaleza, tiene control sobre el daño ambiental ocurrido. El efecto indirecto de la controlabilidad fue parcialmente explicado por la ira sentida. Los efectos directo e indirecto de la responsabilidad, por otro lado, no fueron significativos y se explicaron parcialmente como una manera moderadora en la relación entre la controlabilidad y la discontinuidad del proveedor después del daño ambiental. El estudio identifica el rol conductual de una emoción negativa experimentada durante las crisis gerenciales, influyendo en la toma de decisiones de los individuos con respecto a la discontinuidad del proveedor después del acaecimiento de un daño ambiental.

PALABRAS CLAVE I Compras verdes, discontinuidad del proveedor, controlabilidad, responsabilidad del proveedor, experimento. 


\section{INTRODUÇÃO}

O nivel de conscientização sobre o aquecimento global e outras questões ambientais aumentou significativamente, causando uma preocupação das empresas em comprar de modo sustentável (Chin, Malik, Tat, Sulaiman, \& Choon, 2020). Green Purchasing (GP) induz à compra de matéria-prima sustentável, resultando na escolha de fornecedores que prezem por políticas pró-ambientais (Jabbour, Jabbour, Govindan, Kannan, \& Arantes, 2014; Tseng, Islam, Karia, Fause, \& Afrin, 2019). Estudos recentes revelam que o investimento em GP permite que as empresas aumentem 0 seu desempenho econômico, social e ambiental (Ghosh, 2018; Yook, Choi, \& Suresh, 2017; Yu, Zhang, \& Huo, 2019).

A Sony, por exemplo, construiu uma rede de GP e adquire todos os seus componentes ecologicamente corretos de fornecedores chamados "parceiros ecológicos”, assim como a Volvo Group, que implementou atividades de GP com seus fornecedores para aprimorar o desempenho ambiental e econômico (Yook et al., 2017). Por outro lado, em 2010, a Nestlé sofreu retaliações dos consumidores pelo desempenho insustentável do seu fornecedor de óleo de palma, fazendo com que os consumidores a responsabilizassem pelo dano ambiental causado (Hartmann \& Moeller, 2014).

Profissionais de compras buscam selecionar fornecedores alinhados com aspectos pró-ambientais, por meio de GP (Foo, Kanapathy, Zailani, \& Shaharudin, 2019). O dano ambiental causado pelo efeito de questões de responsabilidade ou controlabilidade, além de prejudicar o meio ambiente, afeta o relacionamento entre comprador e fornecedor. Assim, esta pesquisa tem como objetivo analisar os efeitos direto e indireto da controlabilidade e da responsabilidade na descontinuidade do fornecedor após dano ambiental.

Estudos anteriores não analisaram possíveis efeitos diretos e indiretos da controlabilidade e da responsabilidade, mediados por anger (uma emoção/reação negativa), na descontinuidade do fornecedor após a ocorrência de um dano ambiental no contexto de GP. Harth, Leach e Kessler (2013) examinaram os efeitos de anger de um grupo de indivíduos em tipos de comportamento ambiental e mostraram que enquadrar o grupo como responsável por danos ambientais aumentou a culpa e anger sentida. Polyviou, Rungtusanatham, Reczek e Knemeyer (2018) analisaram os efeitos diretos e indiretos, mediados por anger, da controlabilidade e da responsabilidade na interrupção de fornecimento de componentes críticos. 0 presente estudo encontra espaço na literatura e difere-se do estudo de Polyviou et al. (2018) por acrescentar aspectos sustentáveis no seu contexto, analisando a quantidade de anger sentida e seu efeito na descontinuidade do fornecedor após um dano ambiental causado, diferente do estudo anterior, que enfocou os efeitos da descontinuidade de fornecimento.

\section{REFERENCIAL TEÓRICO E DESENVOLVIMENTO DE HIPÓTESES}

\section{Seleção do fornecedor e continuidade}

Práticas de GP englobam selecionar fornecedores que possuam materiais e serviços ecológicos (Chin et al., 2020). Para isso, é necessário repensar os critérios de aquisição e seleção de materiais e o desenvolvimento de fornecedores (Garzon, Enjolras, Camargo, \& Morel, 2019; Teixeira, Jabbour, Jabbour, Latan, \& Oliveira, 2016). Isso envolve a gestão ativa em todos os aspectos do produto, desde a matéria-prima até o correto descarte.

Selecionar os fornecedores é uma das tarefas essenciais da compra, reduzindo o risco e maximizando o valor geral para a empresa (Chin et al., 2020). Além disso, fortalece a estrutura social e econômica da empresa, 
ao minimizar seus custos e garantir a satisfação do consumidor (Zimmer, Fröhling, \& Schultmann, 2016). As empresas têm buscado cada vez mais incorporar fornecedores sustentáveis em suas operações, visando o desempenho ambiental e sua imagem corporativa (Fong, García-Alcaraz, Maldonado, Ramírez, \& Loya, 2019).

Os fatores associados a impactos negativos que afetam o meio ambiente durante o processo de produção e operação aumentaram significativamente (Rao, Goh, \& Zheng, 2017). Com isso, os consumidores têm se preocupado mais com a compra de produtos sustentáveis, tornando o relacionamento das empresas com fornecedores parte vital do gerenciamento da cadeia de suprimentos, sendo necessário adotar critérios sustentáveis para uma cadeia de suprimentos "verde" (Ji, Ma, \& Li, 2015). O primeiro passo para proteger o meio ambiente e implementar a cadeia de suprimentos "verde" é selecionar fornecedores e comprar matérias-primas sustentáveis (Kannan, Mina, Nosrati-Abarghooee, \& Khosrojerdi, 2020). Os fornecedores desempenham papel fundamental na implementação de iniciativas sustentáveis da cadeia de suprimento e na obtenção de ganhos sociais, ambientais e econômicos (Luthra, Govindan, Kannan, Mangla, \& Garg, 2017).

Na seleção do fornecedor, é importante determinar os riscos inerentes à continuidade do fornecimento (Tsai, 2016). A descontinuidade do fornecedor devido a desastres naturais ou provocados pelo homem é um evento com baixa probabilidade de ocorrência, porém possui impactos negativos (Sawik, 2014). Um exemplo é o caso da Ericsson. Um incêndio na fábrica de um de seus fornecedores de microchips (Philips) destruiu uma quantidade grande de componentes eletrônicos, além de causar um sério dano ambiental, fazendo com que a Ericsson sofresse uma perda aproximada de 400 milhões de euros (Meena \& Sarmah, 2016). Quando um fornecedor é responsabilizado por ações que poderiam ser controláveis, é mais provável que os clientes experimentem anger e ocorra maior probabilidade de retaliação (Folkes, 1984; Sung \& Yih, 2019). Assim, incidentes negativos - como os danos ambientais - podem ser percebidos como fatores determinantes da dependência da empresa em relação ao fornecedor.

\section{Controlabilidade e descontinuidade do fornecedor após dano ambiental}

A controlabilidade é um fator relevante nas respostas a eventos adversos como a descontinuidade do fornecedor e refere-se à avaliação do indivíduo sobre se a causa do evento é incontrolável ou controlável pelo fornecedor (Folkes, 1984; Nikbin, Hyun, Iranmanesh, Maghsoudi, \& Jeong, 2016). Estudos utilizam o termo controlabilidade para caracterizar fontes de riscos e falhas que uma empresa pode evitar (Choi \& Mattila, 2008; Grégoire \& Fischer, 2006; Nikbin et al., 2016).

No contexto da descontinuidade do fornecedor, controlabilidade designa se o fornecedor poderia ou não ter feito algo para impedir a interrupção no fornecimento (Polyviou et al., 2018). Um incidente insustentável do fornecedor, quando ocorrido por fatores incontroláveis (por exemplo, pela natureza), em oposição aos fatores internos controláveis (por exemplo, falhas e desastres ocasionados pelo fornecedor), leva compradores a atribuírem menos responsabilidade pelo dano causado (Hartmann \& Moeller, 2014). Portanto, a controlabilidade, seja por parte do fornecedor ou pela natureza (Polyviou et al., 2018), possui impacto na descontinuidade do fornecedor e pode produzir respostas negativas dos compradores se eles perceberem que o fornecedor tinha a capacidade de impedir um evento negativo, mas não o fez (Nikbin et al., 2016). Assim, a seguinte hipótese é sugerida:

H1: Quando o dano ambiental pode ser controlado pelo fornecedor, o efeito na descontinuidade das compras é significante.

\section{Responsabilidade e descontinuidade do fornecedor após dano ambiental}


A responsabilidade pode ser definida como a medida em que alguma pessoa, ao tomar uma decisão, apresenta um senso de propriedade do resultado, dando crédito a si mesma por bons resultados e culpando-se pelos maus (Botti \& McGill, 2006). Os indivíduos são julgados responsáveis pela ocorrência de algum evento, de ações que ocorreram ou que poderiam ter sido previstas (Munyon, Jenkins, Crook, Edwards, \& Harvey, 2019). No contexto deste estudo, a responsabilidade refere-se à tarefa de seleção de fornecedor que ocorreu antes do dano ambiental. Diz respeito ao fato de o responsável pelas compras ou outra pessoa ter recomendado o fornecedor (Polyviou et al., 2018). Portanto, a ocorrência do evento pode ser considerada devido à responsabilidade de outra pessoa ou de quem atualmente exerce a atividade (Frijda, 1987).

Responsabilidade pode introduzir vieses nas decisões de compras após o dano ambiental. Acredita-se que quando é o responsável pelas compras que recomendou o fornecedor, maior será a probabilidade de continuar com esse fornecedor após a interrupção no fornecimento, devido a gastos com recursos financeiros e humanos, bem como ao tempo na busca, avaliação e seleção de um novo fornecedor (Polyviou et al., 2018). Por outro lado, é menos provável que o responsável pelas compras que não tenha recomendado o fornecedor mantenha relacionamento após o dano ambiental, já que não investiu recursos no fornecedor e, portanto, pode estar menos comprometido. Dessa forma, a probabilidade de um responsável pelas compras não continuar com o fornecedor após um dano ambiental é maior quando outra pessoa, e não ele, o recomendou. Assim, as seguintes hipóteses são sugeridas:

H2: $\mathrm{O}$ efeito do dano ambiental na descontinuidade de compras é maior quando o fornecedor foi selecionado por um terceiro do que quando pelo atual gestor da área.

H3: A responsabilidade pela seleção do fornecedor modera a relação entre a controlabilidade do dano ambiental e a descontinuidade do fornecedor.

\section{Anger}

No ramo da Psicologia, as emoções geralmente são divididas em negativas, como raiva, medo, culpa, vergonha, tristeza e repulsa, e em emoções positivas, como felicidade, orgulho, amor e alívio, bem como alguns fenômenos emocionais, como esperança e compaixão (Lazarus, 1991). A raiva (anger), por exemplo, é considerada uma das emoções negativas mais poderosas de impacto nas relações sociais (Lazarus, 1991) e nos processos e resultados organizacionais (Kumar, Kleef, \& Higgins, 2019). Anger é frequentemente experimentada durante crises gerenciais (Lerner \& Tiedens, 2006) e influencia interpretações, julgamentos e tomadas de decisões de indivíduos na percepção de controlabilidade e responsabilidade após a ocorrência de eventos negativos (Lerner \& Keltner, 2000; Lerner \& Tiedens, 2006). Harth et al. (2013) indicaram que a responsabilidade de um grupo de indivíduos por causar danos ambientais deve levar a menos orgulho, mas maior culpa e anger. Hartmann e Moeller (2014) enfatizam que reações individuais a incidentes negativos sobre questões ambientais são de natureza emocional e comportamental, de modo que os compradores expressam anger por uma organização que responsabilizam, influenciando, assim, a sua decisão de compra. Da mesma forma, Liang, Hou, Jo e Sarigöllü (2019) afirmam que as pessoas sentem anger pelo comportamento ambientalmente irresponsável de uma empresa, pelos efeitos causados.

Uma das principais dimensões da avaliação que distingue anger nos contextos de consumo é a responsabilidade ou o alto controle pelo evento negativo (Sung \& Yih, 2019). Anger influencia as interpretações situacionais mais básicas para o julgamento e a tomada de decisão: as percepções de controle e responsabilidade, permanecendo mesmo após os eventos críticos (Lerner \& Tiedens, 2006). Com a ocorrência de incidentes negativos por falha do fornecedor, devido a ações de controlabilidade e responsabilidade, compradores experienciam anger 
e, provavelmente, reavaliam a continuidade com esse fornecedor (Vidal, 2014). Quando incidentes críticos negativos são atribuídos a ações controláveis pelo fornecedor, é mais provável que os compradores experimentem anger como uma emoção negativa sobre o evento causado (Folkes, 1984; Nikbin et al., 2016). Já quando a responsabilidade é de outra pessoa, é provável que anger aumente devido ao evento causado (Rummelhagen \& Benkenstein, 2017). Portanto, sugerem-se as seguintes hipóteses:

H4a: $\mathrm{O}$ efeito da controlabilidade na descontinuidade do fornecedor após o dano ambiental é mediado pela quantidade de anger sentida.

H4b: $O$ efeito da responsabilidade na descontinuidade do fornecedor após o dano ambiental é mediado pela quantidade de anger sentida.

A partir das hipóteses formuladas, apresenta-se o modelo conceitual da pesquisa, destacando-se os efeitos diretos e indiretos da controlabilidade e da responsabilidade, mediados por anger, na descontinuidade do fornecedor após o dano ambiental (Figura 1).

Figura 1. Modelo teórico

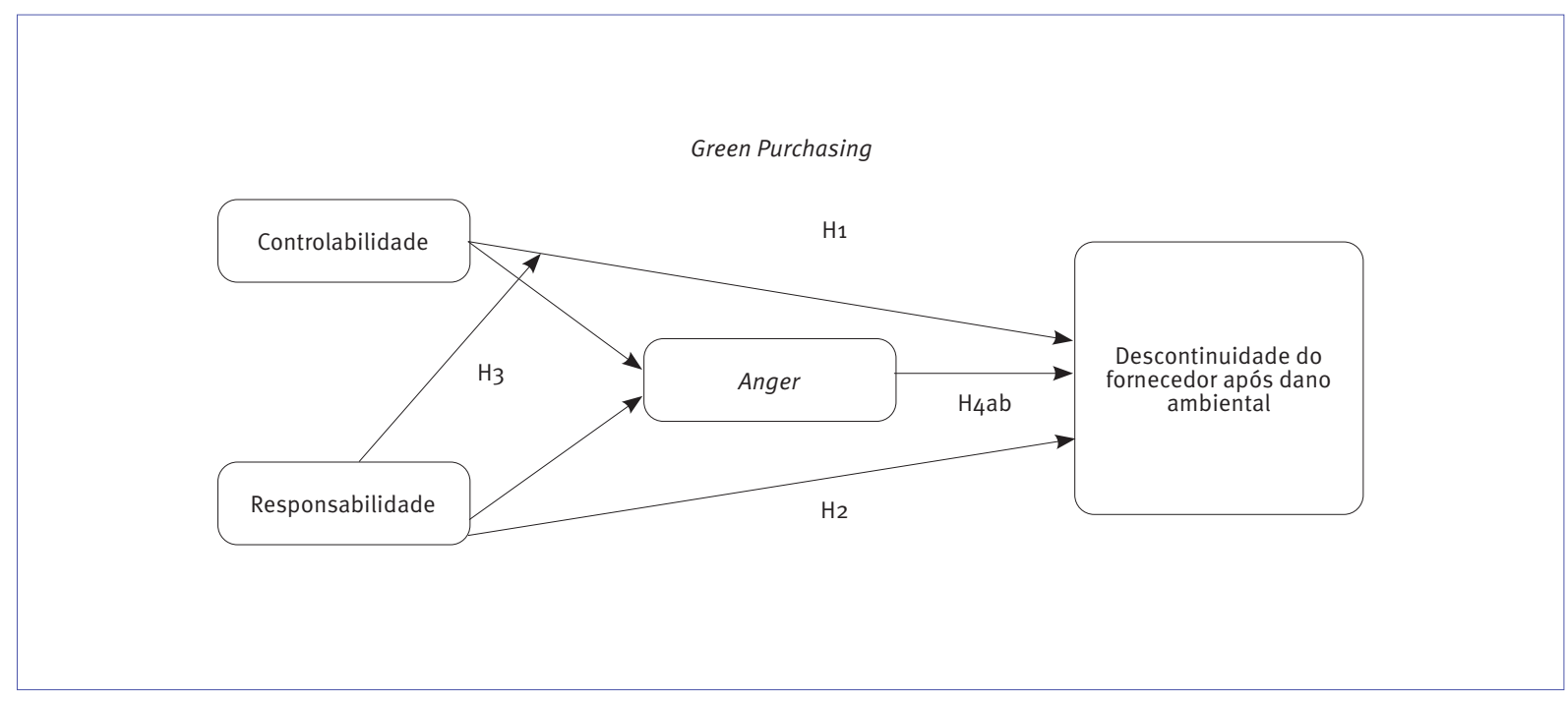

Fonte: Adaptado de Polyviou et al. (2018),

\section{PROCEDIMENTOS METODOLÓGICOS}

De modo a testar as hipóteses do estudo, foi realizado um experimento baseado em cenários (Rungtusanatham, Wallin, \& Eckerd, 2011). Esse método é indicado para o estudo de julgamentos, preferências e decisões humanas que envolvem fenômenos complexos que são difíceis de observar em tempo real (Chen, Rungtusanatham, \& Goldstein, 2019; Eckerd, 2016), como é o caso deste estudo - que pretende verificar, no contexto de GP, os efeitos diretos e indiretos da manipulação da controlabilidade e da responsabilidade na descontinuidade do fornecedor, após uma situação de dano ambiental. 


\section{Desenho das vinhetas}

Os dados foram coletados usando um desenho fatorial $2 \times 2$ (between subjects), cujas variáveis manipuladas (controlabilidade: da natureza e do fornecedor; e responsabilidade: própria e de outra pessoa) envolveram quatro diferentes vinhetas e um módulo comum (descrevendo a situação em torno do experimento). As vinhetas foram previamente validadas em língua inglesa no estudo de Polyviou et al. (2018) e adaptadas para o contexto de GP, sendo revisadas por três profissionais da área de operações e cadeia de suprimento, dos quais um era nativo da língua inglesa. Não foi necessária a realização do pré-teste das vinhetas, consoante as pesquisas de Ro, Su e Chen (2016) e Chen, Ro e Su (2014), que similarmente adaptaram vinhetas validadas de outros estudos sobre a interrupção da relação entre comprador-fornecedor.

\section{Estímulos para controlabilidade e responsabilidade}

As vinhetas incluem descrições para os dois fatores experimentais de controlabilidade e responsabilidade adaptados de Polyviou et al. (2018). Para controlabilidade, descreve-se uma situação de desastre ambiental ocorrido na empresa fornecedora Thai Electronic, sob o controle da natureza ou sob o controle do fornecedor. Para responsabilidade, descreve-se qual dos dois indivíduos na figura de diretor de compras da Reliable Digital, o antecessor (responsabilidade de outra pessoa) ou o atual (responsabilidade própria), havia liderado a equipe de compras, avaliando e recomendando a empresa Thai Electronic à alta gerência da Reliable Digital como a principal fornecedora.

\section{Variáveis dependentes}

Ao final de cada vinheta descritiva, os sujeitos deparavam-se com questões sobre as variáveis dependentes do estudo: anger e descontinuidade do fornecedor após o dano ambiental. Para a variável anger, após a leitura da vinheta, era solicitado ao respondente que determinasse o quanto de anger sentiu após saber sobre o dano ambiental causado pela fornecedora Thai Eletronic, sendo a variável operacionalizada em uma escala do tipo Likert de 7 pontos, variando de 1 para "nem um pouco" a 7 "extremamente". Para avaliar a descontinuidade do fornecedor após o dano ambiental, ao final da leitura da vinheta, o sujeito deveria tomar a decisão de o quão provável seria manter a Thai Eletronic como a principal fornecedora de um de seus componentes eletrônicos, também operacionalizada em uma escala do tipo likert de 7 pontos, variando de 1 para "pouco provável” a 7 "muito provável”.

\section{Definição e coleta da amostra}

Os sujeitos da pesquisa foram obtidos por meio da plataforma on-line Prolific (www.prolific.co), a qual vem sendo utilizada por muitos pesquisadores no recrutamento de sujeitos para experimentos em ciências sociais e econômicas (Palan \& Schitter, 2018). Por exemplo, na área de operações, Nunes, Park e Paiva (2020) utilizaram a plataforma Prolific para recrutar os participantes. Os sujeitos do estudo são profissionais, com experiência em gestão, nativos dos Estados Unidos (EUA) e maiores de 18 anos.

A amostra final foi composta por 267 participantes, sendo 134 do sexo feminino (50,02\%), 132 do sexo masculino $(49,04 \%)$ e um não informado $(0,4 \%)$. A média de idade dos sujeitos foi de $37,33( \pm 11,59)$ anos, cuja familiaridade com a área de compras ou gestão da cadeia de suprimento apresentou média igual a 4,16 $( \pm 1,31)$ - em uma escala do tipo Likert de 7 pontos, variando de 1 para "sim" a 7 para "não" - e média 4,01 $( \pm 1,32)$ para as respostas de familiaridade com danos ambientais na cadeia de suprimento (usando a mesma escala). Poste- 
riormente, verificou-se a adequação do tamanho da amostra, por meio do software G*Power 3.1.9.4. Para isso, foram observados os seguintes parâmetros: (a) o poder estatístico recomendado $(0,80)$; (b) o tamanho do efeito $(f=0,25) ;(c)$ o total de grupos $(2 \times 2=4)$; e (d) os graus de liberdade do numerador $(2-1=1)$. A amostra mínima calculada foi estimada em 128 casos, indicando que o tamanho da amostra neste estudo é adequado. Um elevado poder estatístico garante que uma relação não significativa $(p>0,05)$ seja realmente refutada no estudo, evitando, dessa forma, a ocorrência do erro tipo 2 (falso negativo) (Steiger, 2009).

\section{Procedimentos do experimento}

Os 270 sujeitos foram direcionados aleatoriamente (random assignment) a uma das quatro condições experimentais, por meio do A/B teste provido pelo SurveyMonkey Inc. Ao se depararem com as vinhetas, os participantes deveriam assumir o papel de Diretor de Compras da Reliable Digital. Enquanto realizavam a leitura, algumas questões de verificação de atenção sobre as informações fornecidas nas vinhetas descritivas foram feitas aos participantes. Ao final da leitura, eles deveriam responder às questões referentes à descontinuidade do fornecedor após o dano ambiental, e sobre anger. 0 experimento finalizou coletando as respostas sobre as verificações experimentais (verificações de realismo e manipulação) e os dados demográficos dos sujeitos.

\section{Verificações do experimento}

Duas questões adaptadas de Polyviou et al. (2018) foram respondidas com a intenção de verificar a atenção dos sujeitos, a primeira questionando o papel do respondente no contexto da vinheta (se naquela situação era o Gerente de Recursos Humanos ou o Diretor de Compras), e a segunda perguntando ao sujeito se a empresa Thai Eletronic fornecia discos rígidos ou borrachas. Em ambos os casos, 98,5\% dos respondentes indicaram a resposta correta. Optou-se pela eliminação dos participantes cujas respostas não foram corretas, gerando uma amostra final de 267 sujeitos.

Para verificar o realismo do experimento, duas questões adaptadas do estudo de Polyviou et al. (2018) foram utilizadas, ambas operacionalizadas em escala tipo Likert de sete pontos, variando de 1 para "discordo totalmente" a 7 para "concordo totalmente”. A primeira questão, perguntando se "esse cenário é realístico", obteve média 4,99 $( \pm 1,43)$ e a segunda questão, "assumi seriamente o papel designado como Diretor de Compras da Reliable Digital”, obteve média 6,25 ( $\pm 1,10)$, evidenciando que os participantes consideraram os cenários realistas.

Embora o presente estudo tenha utilizado as vinhetas previamente validadas, foi mantido o teste de verificação de manipulação. Para a variável controlabilidade, solicitou-se aos sujeitos que avaliassem até que ponto o dano ambiental causado pela empresa Thai Electronic estava sob controle da natureza ou do fornecedor, em uma escala de resposta do tipo Likert de sete pontos, variando de 1 para "o fornecedor estava controlando" a 7 "a natureza estava controlando". Os resultados da ANOVA indicaram diferenças significativas entre os sujeitos designados para cada um dos grupos $\left(F_{1,265}=130.651 ; p<0,000\right)$, cuja média do "controle do fornecedor" foi 2,62 $( \pm 1,72)$ e do "controle da natureza" foi 5,09 $( \pm 1,80)$. Para a variável responsabilidade, solicitou-se aos sujeitos que indicassem quem eles achavam que era o responsável por avaliar e recomendar a fornecedora Thai Eletronic, usando uma escala de resposta do tipo Likert de sete pontos (1: “Eu era responsável” a 7: "Outra pessoa era responsável"). Os resultados da ANOVA também indicaram diferenças significativas entre os sujeitos em cada grupo $\left(F_{1,265}=362.787 ; \mathrm{p}<0,000\right)$, cuja média da "responsabilidade própria" foi 1,70 $( \pm 1,59)$ e da "responsabilidade de outra pessoa" foi $5,79( \pm 1,91)$. 


\section{ANÁLISE DOS RESULTADOS}

\section{Variáveis de controle}

Os resultados das variáveis de controle (Tabela 1) gênero, idade, familiaridade com compras/gestão da cadeia de suprimento (Familiaridade 1) e familiaridade com danos ambientais na cadeia de suprimento (Familiaridade 2) apresentaram apenas a variável idade como negativa e significante $(b=-0,02 ; p<0,05)$, indicando que quanto menor a idade do participante, maior a anger sentida (Modelo I). No Modelo II, somente a variável "Familiaridade 2" mostrou ser positiva e significante $(b=0,28 ; p<0,01)$, sugerindo que quando os sujeitos possuem maior familiaridade com algum dano ambiental na cadeia de suprimentos, maior é a probabilidade de continuar com o fornecedor atual.

Tabela 1. Resultados da Regressão Múltipla

\begin{tabular}{|c|c|c|c|c|}
\hline \multirow{3}{*}{ Variáveis dependentes } & \multicolumn{2}{|c|}{ Modelo I } & \multicolumn{2}{|c|}{ Modelo II } \\
\hline & \multicolumn{2}{|c|}{ Anger } & \multicolumn{2}{|c|}{ Continuidade do fornecedor } \\
\hline & $b$ & SE & $\mathrm{b}$ & SE \\
\hline (Constante) & $5,4186^{\star * \star}$ & 0,5676 & $3,1159^{\star \star \star}$ & 0,5778 \\
\hline \multicolumn{5}{|l|}{ Variáveis experimentais } \\
\hline Controlabilidade & $1,1886^{\star \star \star}$ & 0,2142 & $-0,5291^{\star \star}$ & 0,1984 \\
\hline Responsabilidade & $-0,3567$ & 0,2138 & 0,3134 & 0,1882 \\
\hline \multicolumn{5}{|l|}{ Interações } \\
\hline Controlabilidade $\mathrm{x}$ responsabilidade & 0,5685 & 0,4279 & $-0,3854$ & 0,3759 \\
\hline \multicolumn{5}{|l|}{ Mediador } \\
\hline Anger & - & - & $-0,2266^{\star \star \star}$ & 0,0544 \\
\hline \multicolumn{5}{|l|}{ Variáveis de controle } \\
\hline Gênero & $-0,3819$ & 0,2125 & 0,0470 & 0,1872 \\
\hline Idade & $-0,0221^{\star}$ & 0,0094 & $-0,0036$ & 0,0083 \\
\hline Familiaridade 1 & 0,0633 & 0,1060 & $-0,0553$ & 0,0929 \\
\hline Familiaridade 2 & 0,0489 & 0,1023 & $0,2763^{\star *}$ & 0,0896 \\
\hline$R$ & \multicolumn{2}{|c|}{0,3774} & \multicolumn{2}{|c|}{0,4144} \\
\hline$R^{2}$ & \multicolumn{2}{|c|}{0,1424} & \multicolumn{2}{|c|}{0,1717} \\
\hline$R^{2}$ change & \multicolumn{2}{|c|}{0,0058} & \multicolumn{2}{|c|}{0,0034} \\
\hline F-statistic & \multicolumn{2}{|c|}{$1,7651^{\star \star *}$} & \multicolumn{2}{|c|}{$1,0512^{\star \star \star}$} \\
\hline N & \multicolumn{2}{|c|}{267} & \multicolumn{2}{|c|}{267} \\
\hline
\end{tabular}

Notas: Significância ao nível de * p<0,05; ** p<0,01; *** p<0,001.

Modelos estimados utilizando intervalo de confiança com 10.000 bootstrap. 


\section{Efeitos da controlabilidade, da responsabilidade e de anger na descontinuidade do fornecedor}

Para verificar as hipóteses, foi realizada análise de regressão múltipla utilizando a macro Process (Hayes, 2018). Os resultados do Modelo II (Tabela 1) apresentam que anger possui efeito negativo e significante na descontinuidade do fornecedor $(b=-0,23 ; \mathrm{p}<0,001)$. Quanto mais anger sentida, menor é a probabilidade de continuar com o fornecedor após o dano. 0 resultado do efeito direto da controlabilidade na continuidade do fornecedor é negativo e significante $(b=-0,53 ; \mathrm{p}<0,01)$. Quando o dano ambiental causado está sob controle do fornecedor, maior é a probabilidade de descontinuidade, suportando a hipótese $\mathrm{H}_{1}$. Já o efeito direto da responsabilidade na continuidade do fornecedor é positivo e não significante $(b=0,31 ; \mathrm{p}=0,09)$, não confirmando a hipótese $\mathrm{H} 2$. A probabilidade de descontinuidade após o dano ambiental é maior quando quem o selecionou foi outra pessoa, porém a relação não se mostrou significante no modelo.

A interação entre a controlabilidade e a responsabilidade na continuidade do fornecedor apresentou uma relação negativa e não significante $(b=-0,39 ; p=0,38)$. A análise de efeito condicional da responsabilidade na relação entre controlabilidade e descontinuidade do fornecedor (Tabela 2), por meio da técnica Johnson-Neyman, apresenta que quando a responsabilidade é própria, o efeito é negativo e significante $(b=-0,72 ; p=0,01)$. Já quando a responsabilidade é de outro, o efeito permanece negativo, mas não significante $(b=-0,34 ; p=0,22)$, suportando, portanto, parcialmente a hipótese $\mathrm{H}_{3}$.

Tabela 2. Efeitos condicionais moderador da responsabilidade

\begin{tabular}{l|c|c|c|c|c}
\hline Responsabilidade & Efeito & Se & t & ULCI \\
\hline$-0,5000$ & $-0,3364$ & 0,2759 & $-1,2193$ & 0,2239 & $-0,8797$ \\
\hline 0,5000 & $-0,7218^{*}$ & 0,2707 & $-2,6670$ & 0,2069 & $-1,2548$ \\
\hline
\end{tabular}

Nota: Significância ao nível de * p<0,01

Legenda: $-0,5=$ Responsabilidade de outra pessoa; 0,5 = Responsabilidade própria.

0 efeito indireto da controlabilidade na continuidade do fornecedor via anger é negativo e significante $(b=-0,27 ; p<0,01)$, sugerindo que os sujeitos, quando percebem que o dano ambiental era de controle do fornecedor, sentem mais raiva, e é mais provável que descontinuem o fornecedor. Entretanto, esse efeito indireto da controlabilidade é menos intenso quando comparado ao seu efeito direto na continuidade do fornecedor ( $b$ $=-0,53 ; p<0,01)$, suportando parcialmente a hipótese H4a. Dessa forma, anger é uma condição presente, mas não necessária, para a decisão de descontinuidade do fornecedor perante o dano.

Já a responsabilidade não possui efeito significante em anger ( $b=-0,36 ; p=0,09)$, nem efeito significante direto na continuidade do fornecedor, não dando suporte à hipótese $\mathrm{H}_{4}$ b. Portanto, identificou-se que a responsabilidade não possui influência direta ou indireta na continuidade do fornecedor.

\section{DISCUSSÃO E IMPLICAÇÕES}

Os resultados do efeito da controlabilidade na descontinuidade do fornecedor após o dano ambiental confirmam a hipótese $\mathrm{H}_{1}$, fornecendo evidências teóricas e empíricas de que quando o dano ambiental causado está sob controle do fornecedor e não da natureza, menor é a probabilidade de manter esse fornecedor. Quando o comprador acredita 
que um evento poderia ser evitado por meio da controlabilidade do fornecedor, a probabilidade de descontinuidade é maior (Nikbin et al., 2016). Polyviou et al. (2018) identificaram que a probabilidade da descontinuidade do fornecedor após a interrupção de fornecimento é maior quando o gestor de compras percebe que o fornecedor, e não a natureza, tinha controle sobre o evento de interrupção. Portanto, consoante os resultados de Polyviou et al. (2018), o presente estudo identificou que quando o dano ambiental causado poderia ser controlado pelo fornecedor, não sendo, portanto, causado pela natureza, maior é a probabilidade de descontinuidade. Compradores atribuem maior responsabilidade pelo comportamento insustentável do fornecedor quando o incidente negativo poderia ter sido controlado nos locais internos de fabricação da empresa, ao invés de situações de ocorrências externas, como eventos naturais (Hartmann \& Moeller, 2014).

Os resultados associados à controlabilidade indicam que os indivíduos reagem de maneira negativa quando acreditam que o fornecedor poderia ter evitado um evento negativo e não o faz (Choi \& Mattila, 2008; Nikbin et al., 2016), o que pode levar à descontinuidade do fornecedor após um dano ambiental causado. Em eventos como a descontinuidade no fornecimento, os Gerentes de Compras não atribuem responsabilidade ao fornecedor quando o evento é incontrolável (Timmer \& Kaufmann, 2019). Entretanto, como destacado por Grégoire e Fischer (2006), quando a empresa é responsável por uma falha que poderia ter sido controlada e evitada, é provável que haja retaliação por parte do comprador devido às consequências negativas. Assim, o presente estudo observou que a falta de controlabilidade do fornecedor sobre o dano ambiental causado é um fator decisivo e influenciador na descontinuidade do relacionamento do gestor de compras com o fornecedor.

Já o efeito da responsabilidade na descontinuidade do fornecedor após o dano ambiental não apresentou significância direta. Esses resultados vão ao encontro ao estudo de Polyviou et al. (2018), os quais identificaram que a descontinuidade do fornecedor não é uma função do líder de recuperação atual, ou mesmo quando outra pessoa ter recomendado, o fornecedor antes da ocorrência da interrupção do fornecimento. Embora a seleção do fornecedor seja uma atividade essencial da compra (Chin et al., 2020) e uma atividade primordial de GP (Garzon et al., 2019), os resultados sugerem que a associação entre a seleção do fornecedor e a sua descontinuidade após o dano ambiental pode não ser explicada pela responsabilidade própria (pelo atual gestor de compras) ou de outra pessoa em ter selecionado o fornecedor envolvido com o dano ambiental. Mesmo considerando o contexto de práticas de GP, que prezam pela seleção de fornecedores que atendam a objetivos sustentáveis, as descobertas do estudo identificam que a responsabilidade (própria ou de outra pessoa) pela indicação e seleção de um fornecedor não influencia de maneira direta a sua descontinuidade após o dano ambiental causado. Esses resultados fornecem evidências contrárias à Teoria da Avaliação da Psicologia, as quais indicam que a responsabilidade por um evento ativa anger (Lerner \& Keltner, 2000), o que, por sua vez, influencia negativamente o comportamento e as decisões do indivíduo.

No entanto, os resultados da responsabilidade como moderadora na relação entre a controlabilidade e a descontinuidade do fornecedor indicaram que quando a responsabilidade de quem recomendou o fornecedor é própria, maior é o efeito da controlabilidade na descontinuidade do fornecedor. Esse resultado não corrobora a premissa de que se a responsabilidade fosse própria, maior seria a probabilidade de manter o fornecedor. Nesse sentido, acredita-se que o gestor se sinta corresponsável pelo dano ambiental. Dessa forma, esse resultado se diferencia dos achados de Polyviou et al. (2018). Nesse sentido, este estudo contribui ao identificar que a responsabilidade do gestor de compras possui influência na tomada de decisão de continuidade com o fornecedor.

Os resultados de anger apresentaram efeitos negativos e significantes na descontinuidade do fornecedor, indicando que quanto mais anger sentida, menor a probabilidade de continuar com o fornecedor após o dano. Os valores ambientais podem influenciar esses comportamentos, uma vez que as pessoas que priorizam 
questões ambientais devem ter mais chances de agir contra incidentes ambientais insustentáveis (Hartmann \& Moeller, 2014; Liang et al., 2019). Os resultados indicaram, ainda, um efeito significante da controlabilidade em anger quando o dano ambiental causado está sob controle do fornecedor, o que aumenta a anger sentida. Os sujeitos no papel de Diretor de Compras sentiram mais anger quando o dano ambiental foi de controle do fornecedor, e não da natureza. Como Nerb e Spada (2001) destacaram, as ações que resultam em alta responsabilidade de um ator, por exemplo, sobre a controlabilidade de um dano, maior é a anger sentida. Anger está associada à tendência comportamental de agir contra um parceiro em uma aliança empresarial, desde meramente expressar desagrado a casos mais extremos, como uma ação legal ou ruptura do relacionamento (Kumar et al., 2019).

Quando a responsabilidade em ter selecionado o fornecedor é de outra pessoa, e não do atual gestor da área, a anger sentida tende a ser maior. Estudos como o de Böhm (2003) indicam que as emoções como anger e indignação são dirigidas a alguém e, portanto, implicam que a responsabilidade é atribuída a outra pessoa. Lerner e Keltner (2000) retratam que anger surge da avaliação de responsabilidade de outra pessoa por eventos negativos, controle individual e um senso de certeza sobre o que aconteceu. Frijda (1987) identificou que a responsabilidade de outra pessoa é passível de anger e que a responsabilidade própria, não.

0 efeito indireto da controlabilidade na descontinuidade do fornecedor via anger apresentou resultado significante, sugerindo que os sujeitos, quando percebem que o dano ambiental poderia ter sido controlado pelo fornecedor, sentem mais anger, sendo subsequentemente mais prováveis a descontinuarem o fornecedor. Entretanto, o efeito indireto de anger na relação entre controlabilidade e descontinuidade do fornecedor é menor quando comparado ao efeito direto da controlabilidade na descontinuidade do fornecedor. Esses resultados vão ao encontro dos achados de Polyviou et al. (2018), os quais indicaram que o efeito da controlabilidade na descontinuidade do fornecedor após a interrupção no fornecimento pode ser parcialmente explicado pela quantidade de anger experimentada devido ao evento de interrupção.

Por fim, os resultados mostram que a descontinuidade do fornecedor após o dano ambiental é baseada não apenas de maneira racional, mas também emocional, por meio de anger. As reações individuais são de natureza emocional e comportamental, de modo que os consumidores expressam anger por uma organização que eles responsabilizam devido a incidentes insustentáveis (Hartmann \& Moeller, 2014).

\section{Implicações teóricas e gerenciais}

Quando a causa do evento insustentável foi devido à falta de controle do fornecedor, e não por evento da natureza (Hartmann \& Moeller, 2014), maior é a probabilidade de não se manter o relacionamento com o fornecedor. Isso implica a aplicação de critérios ambientais na seleção de fornecedores por meio de GP, seja exigindo um comportamento sustentável ou formando um painel de fornecedores comprometidos com suas práticas ambientais no gerenciamento da cadeia de suprimento (Teixeira, Assumpção, Correa, Savi, \& Prates, 2018). Por outro lado, a responsabilidade quanto a quem selecionou o fornecedor que causou o dano ambiental, se o atual gestor da área ou outra pessoa, não influencia diretamente a descontinuidade do fornecedor após o dano ambiental. Embora a ocorrência de algum evento indesejável possa ser considerada devido à responsabilidade de outra pessoa ou de quem atualmente exerce a atividade (Frijda, 1987). Diferente de Polyviou et al. (2018), identificou-se que a responsabilidade própria pela seleção do fornecedor potencializa o efeito da controlabilidade na descontinuidade do fornecedor. Nesse sentido, em caso de dano ambiental, a responsabilidade do gestor em selecionar o fornecedor também pesa na decisão e descontinuidade do fornecedor. 
O estudo também contribui ao analisar o papel comportamental de uma emoção negativa (anger) quando experimentada durante crises gerenciais na cadeia de suprimento sustentável, na decisão de continuidade do fornecedor após a ocorrência de dano ambiental. As pessoas exibem afinidade emocional com a natureza e com o meio ambiente (Liang et al., 2019); emoções negativas como a raiva levam os indivíduos a optarem por sanções punitivas ao responsável (Angie, Connelly, Waples, \& Kligyte, 2011). Como sugerem os resultados, a controlabilidade do evento afeta a emoção negativa do comprador. Quando o dano ambiental poderia ser controlável pelo fornecedor, maior é a anger por parte do comprador. Anger, por sua vez, reduz a probabilidade de continuidade do fornecedor, mostrando-se uma emoção que tem influência na decisão de continuidade. Isso pode gerar consequências que podem levar a outras decisões e ações por parte dos compradores, por exemplo, ações judiciais, impactando negativamente a imagem do fornecedor no mercado e possivelmente outros contratos do fornecedor no mercado, atuais e futuros.

Gerencialmente, o estudo contribui, por meio de cenários que simulam uma situação real do cotidiano organizacional, na tomada de decisão de profissionais de compras no relacionamento com os fornecedores e sua possível descontinuidade após um dano ambiental ocorrido, considerando aspectos de GP na seleção desses agentes e anger como uma emoção negativa após um acidente insustentável. Tendo em vista os resultados da influência da controlabilidade, da responsabilidade e de anger na descontinuidade do fornecedor, a principal implicação gerencial do estudo é que os fornecedores visem uma postura sustentável proativa, primando por práticas sustentáveis, evitando que danos ambientais, em especial os controláveis, possam ocorrer.

Nesse sentido, primeiro, os resultados da variável controlabilidade assinalam que há um efeito direto e significante de um dano ambiental controlável pelo fornecedor na descontinuidade do fornecedor por parte do comprador. Segundo os resultados apontam que a ocorrência de dano ambiental em uma situação que poderia ser controlada pelo fornecedor gera uma emoção negativa no comprador, neste estudo, anger. Essa emoção negativa aumenta a probabilidade de descontinuidade do fornecedor por parte do comprador. E, terceiro, há uma tensão entre controlabilidade e responsabilidade; o efeito da controlabilidade na descontinuidade do fornecedor é potencializado quando o comprador foi responsável pelo desenvolvimento do fornecedor. Isso implica que, possivelmente, um sentimento de corresponsabilidade tenha influência na decisão do comprador em descontinuar o fornecedor. Nesse aspecto específico, maior é a necessidade do fornecedor de desenvolver práticas sustentáveis.

O estudo também destaca a importância no processo de tomada de decisão dos profissionais de compras em relação a fornecedores que se preocupem com a sustentabilidade em suas operações, visto que esse é um dos inúmeros desafios na transição para uma economia circular (Sharma \& Foropon, 2019). Como indicado por Hopkinson, Zils, Hawkins e Roper (2018), é necessário que fornecedores e profissionais de compras desenvolvam competências e capacidades para gerenciar um modelo de negócios complexo de economia circular global. Para isso, devem levar em consideração atributos de produtos "verdes" de maneira eficaz e eficiente, evitando, assim, danos ao meio ambiente.

\section{CONSIDERAÇÕES FINAIS}

0 estudo analisou os efeitos da controlabilidade (se o fornecedor ou a natureza tinham controle sobre o dano ambiental ocorrido) e responsabilidade (se a responsabilidade em ter selecionado o fornecedor que causou o dano ambiental era do atual gestor da área ou de outra pessoa) na descontinuidade do fornecedor após o dano 
ambiental. Também foi analisado se esses efeitos são mediados pela quantidade de anger sentida pelo gestor de compras devido à descontinuidade do fornecedor após o dano ambiental.

Os resultados referentes à controlabilidade indicaram que a probabilidade de descontinuidade do fornecedor é maior quando o fornecedor, e não a natureza, tem controle sob o dano ambiental; bem como os sujeitos, no papel de Diretor de Compras, sentem mais anger quando o dano ambiental é mais de controle do fornecedor do que da natureza. 0 efeito indireto da controlabilidade é parcialmente explicado pela quantidade de anger experimentada pelo gestor de compras, indicando que anger é uma condição presente, mas não necessária para a descontinuidade do fornecedor após o dano ambiental, bem como o efeito da controlabilidade na descontinuidade do fornecedor é potencializado quando a responsabilidade pela escolha do fornecedor é própria do gestor.

0 estudo limitou-se às análises quantitativas das variáveis controlabilidade, responsabilidade, descontinuidade do fornecedor após o dano ambiental e anger, como uma emoção negativa. 0 trabalho, entretanto, considerou o efeito de uma emoção negativa de maneira individual, focando o papel do gestor de compras de uma empresa, não analisando o efeito em diferentes stakeholders (Dufour, Andiappan, \& Banoun, 2019; Hartmann \& Moeller, 2014). Outras formas de medir anger podem ser utilizadas; por exemplo, Hartmann e Moeller (2014) mediram anger utilizando três itens: o quanto os participantes da pesquisa se sentiam zangados, hostis e ofendidos, e Pulles e Loohuis (2020) consideraram a frustração, anger, irritação e tensão dos participantes.

Por fim, sugerem-se pesquisas futuras, as quais envolvam a replicação de descobertas explorando outros tipos de descontinuidade do fornecedor, como problemas com logística no fornecimento ou incidentes de qualidade. Outras emoções além de anger podem ser analisadas nesse contexto - a exemplo: medo, culpa, vergonha, arrependimento, tristeza, felicidade e euforia, bem como situações em que custos de transação estejam evidentes, laços de longo prazo entre fornecedor e comprador, ativos específicos (tangíveis ou intangíveis), que podem afetar a decisão de continuidade do fornecedor em casos de dano ambiental.

\section{REFERÊNCIAS}

Angie, A. D., Connelly, S., Waples, E. P., \& Kligyte, V. (2011). The influence of discrete emotions on judgement and decisionmaking: A meta-analytic review. Cognition \& Emotion, 25(8), 1393-1422. doi:10.1080/02699931.2010.550751

Böhm, G. (2003). Emotional reactions to environmental risks: Consequentialist versus ethical evaluation. Journal of Environmental Psychology, 23(2), 199-212. doi:10.1016/ so272-4944 (02)00114-7

Botti, S., \& McGill, A. L. (2006). When choosing is not deciding: The effect of perceived responsibility on satisfaction. Journal of Consumer Research, 33(2), 211-219. doi:10.1086/ 506302

Chen, Y. S., Ro, Y., \& Su, H. C. (2014). Mirror, mirror on the wall.. Who's the most opportunistic and compliant of them all? American Journal of Business, 29(1), 43-60. doi: 10.1108/ajb05-2013-0028

Chen, Y., Rungtusanatham, M. J., \& Goldstein, S. M. (2019). Historical supplier performance and strategic relationship dissolution: Unintentional but serious supplier error as a moderator. Decision Sciences, 50(6), 1224-1258. doi:10.1111/ deci.12373
Chin, T. A., Malik, N. F. I. A., Tat, H. H., Sulaiman, Z., \& Choon, T. L. (2020). Green purchasing practices and environmental performance. International Journal of Supply Chain Management, 9(1), 291-297. doi: 10.1108/JMTM-04-2020-0173

Choi, S., \& Mattila, A. S. (2008). Perceived controllability and service expectations: Influences on customer reactions following service failure. Journal of Business Research, 61(1), 24-30. doi:10.1016/j.jbusres.2006.05.006

Dufour, L., Andiappan, M., \& Banoun, A. (2019). The impact of emotions on stakeholder reactions to organizational wrongdoing. European Management Review, 16, 761-779. doi: 10.1111/emre.12141

Eckerd, S. (2016). Experiments in purchasing and supply management research. Journal of Purchasing and Supply Management, 22(4), 258-261. doi:10.1016/j. pursup.2016.08.002

Folkes, V. S. (1984). Consumer reactions to product failure: An attributional approach. Journal of Consumer Research, 10(4), 398-409. doi:10.1086/208978 
Fong, J. R., García-Alcaraz, J. L., Maldonado-Macías, A. A., Ramírez, C. S., \& Loya, V. M. (2019). The Impact of Green Attributes From Suppliers on Supply Chain Performance. In I. Management Association (Ed.), Green Business: Concepts, Methodologies, Tools, and Applications (pp. 1216-1232). IGI Global. doi:10.4018/978-1-5225-7915-1.ch059

Foo, M. Y., Kanapathy, K., Zailani, S. \& Shaharudin, M. R. (2019). Green purchasing capabilities, practices and institutional pressure. Management of Environmental Quality, 30(5), 11711189. doi:10.1108/MEQ-07-2018-0133

Frijda, N. H. (1987). Emotion, cognitive structure, and action tendency. Cognition \& Emotion, 1(2), 115-143. doi:10.1080/02699938708408043

Garzon, F. S., Enjolras, M., Camargo, M., \& Morel, L. (2019). A green procurement methodology based on Kraljic matrix for supplier's evaluation and selection: A case study from the chemical sector. Supply Chain Forum: An International Journal, 20(3), 1-17. doi: 10.1080/16258312.2019.1622446

Ghosh, M. (2018). Determinants of green procurement implementation and its impact on firm performance. Journal of Manufacturing Technology Management, 30(2), 462-482. doi: 10.1108/jmtm-06-2018-0168

Grégoire, Y., \& Fisher, R. J. (2006). The effects of relationship quality on customer retaliation. Marketing Letters, 17(1), 3146. doi:10.1007/s11002-006-3796-4

Harth, N. S., Leach, C. W., \& Kessler, T. (2013). Guilt, anger, and pride about in-group environmental behaviour: Different emotions predict distinct intentions. Journal of Environmental Psychology, 34, 18-26. doi:10.1016/j.jenvp.2012.12.005

Hartmann, J., \& Moeller, S. (2014). Chain liability in multitier supply chains? Responsibility attributions for unsustainable supplier behavior. Journal of Operations Management, 32(5), 281-294. doi:10.1016/j.jom.2014.01.005

Hayes, A. F. (2018). Introduction to mediation, moderation, and conditional process analysis. A regression-based approach. New York, USA: The Guilford Press.

Hopkinson, P., Zils, M., Hawkins, P., \& Roper, S. (2018). Managing a complex global circular economy business model: Opportunities and challenges. California Management Review, 6o(3), 71-94. doi:10.1177/0008125618764692

Jabbour, A. B., Jabbour, C., Govindan, K., Kannan, D., \& Arantes, A. F. (2014). Mixed methodology to analyze the relationship between maturity of environmental management and the adoption of green supply chain management in Brazil. Resources, Conservation and Recycling, 92, 255-267. doi:10.1016/j.resconrec.2014.02.004

Ji, P., Ma, X., \& Li, G. (2015). Developing green purchasing relationships for the manufacturing industry: An evolutionary game theory perspective. International Journal of Production Economics, 166, 155-162. doi:10.1016/j.ijpe.2014.10.009

Kannan, D., Mina, H., Nosrati-Abarghooee, S., \& Khosrojerdi, G. (2020). Sustainable circular supplier selection: A novel hybrid approach using streaming data. Science of The Total Environment, 722(1), 1-13. doi:10.1016/j.scitotenv.2020.137936
Kumar, R., Kleef, G. A. V., \& Higgins, E. T. (2019). How emotions influence alliance relationships: The potential functionality of negative emotions. Organizational Psychology Review, 9(2), 157-183. doi:10.1177/2041386619878837

Lazarus, R. S. (1991). Emotion and adaptation. New York, USA: Oxford University Press.

Lerner, J. S., \& Keltner, D. (2000). Beyond valence: Toward a model of emotion-specific influences on judgement and choice. Cognittion and Emotion, 14(4), 473-493. doi:10.1080/026999300402763

Lerner, J. S., \& Tiedens, L. Z. (2006). Portrait of the angry decision maker: How appraisal tendencies shape anger's influence on cognition. Journal of Behavioral Decision Making, 19(2), 115137. doi:10.1002/bdm.515

Liang, D., Hou, C., Jo, M. S., \& Sarigöllü, E. (2019). Pollution avoidance and green purchase: The role of moral emotions. Journal of Cleaner Production, 210, 1301-1310. doi:10.1016/ j.jclepro.2018.11.103

Luthra, S., Govindan, K., Kannan, D., Mangla, S. K., \& Garg, C. P. (2017). An integrated framework for sustainable supplier selection and evaluation in supply chains. Journal of Cleaner Production, 140(3), 1686-1698. doi:10.1016/j. jclepro.2016.09.078

Meena, P. L., \& Sarmah, S. P. (2016). Supplier selection and demand allocation under supply disruption risks. The International Journal of Advanced Manufacturing Technology, 83(4), 265-274. doi:10.1007/s00170-015-7520-5

Munyon, T. P., Jenkins, M. T., Crook, T. R., Edwards, J., \& Harvey, N. P. (2019). Consequential cognition: Exploring how attribution theory sheds new light on the firm-level consequences of product recalls. Journal of Organizational Behavior, 40(5), 587-602. doi: 10.1002/job.2350

Nerb, J., \& Spada, H. (2001). Evaluation of environmental problems: A coherence model of cognition an emotion. Cognition \& Emotion, 15(4), 521-551. doi:10.1080/02699930126254

Nikbin, D., Hyun, S. S., Iranmanesh, M., Maghsoudi, A., \& Jeong, C. (2016). Airline travelers' causal attribution of service failure and its impact on trust and loyalty formation: The moderating role of corporate social responsibility. Asia Pacific Journal of Tourism Research, 21(4), 355-374. doi:10.1080/10941665.201 5.1048265

Nunes, M. F., Park, C. L., \& Paiva, E. L. (2020). Can we have it all? Sustainability trade-offs and cross-insurance mechanisms in supply chains. International Journal of Operations \& Production Management, ahead-of-print. doi: 10.1108/ IJOPM-12-2019-0802

Palan, S., \& Schitter, C. (2018). Prolific.ac a subject pool for online experiments. Journal of Behavioral and Experimental Finance, 17, 22-27. doi:10.1016/j.jbef.2017.12.004

Polyviou, M., Rungtusanatham, M. J., Reczek, R. W., \& Knemeyer, A. M. (2018). Supplier non-retention post disruption: What role does anger play? Journal of Operations Management, 61, 1-14. doi:10.1016/j.jom.2018.07.001 
Pulles, N. J., \& Loohuis, R. P. (2020). Managing buyer-supplier conflicts: The effect of buyer openness and directness on a supplier's willingness to adapt. Journal of Supply Chain Management, 56(4), 65-81. doi.org/10.1111/jscm.12240

Rao, C., Goh, M., \& Zheng, J. (2017). Decision mechanism for supplier selection under sustainability. International Journal of Information Technology \& Decision Making, 16(1), 87-115. doi:10.1142/So219622016500450

Ro, Y. K., Su, H. C., \& Chen, Y. S. (2016). A tale of two perspectives on an impending supply disruption. Journal of Supply Chain Management, 52(1), 3-20. doi:10.1111/jscm.12100

Rummelhagen, K., \& Benkenstein, M. (2017). Whose fault is it? European Journal of Marketing, 51(11/12), 1856-1875. doi:10.1108/EJM-01-2016-0014

Rungtusanatham, M., Wallin, C., \& Eckerd, S. (2011). The vignette in a scenario-based role-playing experiment. Journal of Supply Chain Management, 47(3), 9-16. doi:10.1111/j.1745493X.2011.03232.X

Sawik, T. (2014). Joint supplier selection and scheduling of customer orders under disruption risks: Single vs. dual sourcing. Omega, 43, 83-95. doi:10.1016/j.omega.2013.06.007

Sharma, A., \& Foropon, C. (2019). Green product attributes and green purchase behavior. Management Decision, 57(4), 10181042. doi:10.1108/MD-10-2018-1092

Steiger, J. (2009). GPOWER tutorial. Recuperado de http://www. statpower.net/Content/312/Handout/gpower-tutorial.pdf

Sung, B., \& Yih, J. (2019). The direct and indirect effects of anger and its cognitive appraisals in public relations incidents. Asia Pacific Journal of Marketing and Logistics, 31(5), 1344-1358. doi:10.1108/APJML-08-2018-0292

Teixeira, A. A., Jabbour, C. J. C., Jabbour, A. B. L. S., Latan, H., \& Oliveira, J. H. C. (2016). Green training and green supply chain management: Evidence from Brazilian firms. Journal of Cleaner Production, 116, 170-176. doi:10.1016/j.jclepro.2015.12.061
Teixeira, C. R. B., Assumpção, A. L., Correa, A. L., Savi, A. F., \& Prates, G. A. (2018). The contribution of green logistics and sustainable purchasing for green supply chain management. Independent Journal of Management \& Production, 9(3), 1002-1026. doi:10.1 4807/ijmp.v9i3.789

Timmer, S., \& Kaufmann, L. (2019). Do managers' dark personality traits help firms in coping with adverse supply chain events? Journal of Supply Chain Management, 55(4), 67-97. doi: $10.1111 /$ jscm.12212

Tsai, W. C. (2016). A dynamic sourcing strategy considering supply disruption risks. International Journal of Production Research, 54(7), 2170-2184. doi:10.1080/00207543.20 15.1129465

Tseng, M. L., Islam, M. S., Karia, N., Fauzi, F. A., \& Afrin, S. (2019). A literature review on green supply chain management: Trends and future challenges. Resources, Conservation and Recycling, 141, 145-162. doi:10.1016/j.resconrec.2018.10.009

Vidal, D. (2014). Eye for an eye: Examining retaliation in businessto-business relationships. European Journal of Marketing, 48(1/2), 47-67. doi:10.1108/EJM-03-2011-0173

Yook, K. H., Choi, J. H., \& Suresh, N. C. (2017). Linking green purchasing capabilities to environmental and economic performance: The moderating role of firm size. Journal of Purchasing and Supply Management, 24(4), 326-337. doi:10.1016/j.pursup.2017.09.001

Yu, Y., Zhang, M., \& Huo, B. (2019). The impact of supply chain quality integration on green supply chain management and environmental performance. Total Quality Management \& Business Excellence, 30(9), 1110-1125. doi:10.1080/1478336 3.2017 .1356684

Zimmer, K., Fröhling, M., \& Schultmann, F. (2016). Sustainable supplier management: A review of models supporting sustainable supplier selection, monitoring and development. International Journal of Production Research, 54(5), 14121442. doi:10.1080/00207543.20 15.1079340

\section{CONTRIBUIÇÃO DE AUTORIA}

Josefer de Lima Souza, Vilmar Antonio Gonçalves Tondolo e Rosana da Rosa Portella Tondolo trabalharam na conceitualização e abordagem teórica-metodológica. A revisão teórica foi conduzida por Josefer de Lima Souza. A coleta de dados foi coordenada pelo Vilmar Antonio Gonçalves Tondolo. Josefer de Lima Souza, Vilmar Antonio Gonçalves Tondolo, Rosana da Rosa Portella Tondolo, Guillherme Lerch Lunardi e Flávio Régio Brambilla participaram da análise de dados, da redação e revisão final do manuscrito. 